\title{
THE VECTORCARDIOGRAM IN 100 HEALTHY SUBJECTS USING A NEW DRAWING INSTRUMENT
}

\author{
BY \\ JOHN SHILLINGFORD AND WALLACE BRIGDEN
}

From the Cardiac Department of the London Hospital

Received December 20, 1950

The development of electrocardiography has led to the recording of the potentials produced by the heart at an increasing number of sites on the body surface by various combinations of leads. This trend has focussed attention on greater numbers of patterns, with emphasis on more and more detail. Pari passu with these developments, many investigators have endeavoured to record and interpret the central electrical field of the heart as a whole.

Mann (1920) showed that a single continuous curve could be obtained by the fusion of three leads into a monocardiogram. He constructed the curve from direct voltage measurements at equal and successive time intervals from standard leads ; simultaneous QRS complexes were not measured, but Mann considered that this did not introduce a serious error. Eighteen years later, Mann (1938) described a moving-coil galvanometer that could respond in two planes to the potentials from two leads, and the resulting loop was recorded photographically. This technique has not been generally accepted.

Schellong (1936) and Wilson and Johnston (1938) independently showed that the cathode-ray tube could be used to produce a continuous curve from the amplified voltage of extremity leads; they preferred to call this loop a vectorcardiogram. Wilson and Johnston and others employed leads based on the Einthoven triangle to record the vectorcardiogram in the frontal plane, and more recently a central terminal placed at the back has been used to provide reference in depth to form a tetrahedral system.

Duchosal and Sulzer (1949) have made extensive contributions to this subject using cathoderay apparatus with a split electron beam, thus enabling simultaneous loops from the frontal and saggital planes to be recorded. They have used a parallelepiped reference system.

The slow development of vectorcardiography has been in some measure due to the elaborate and time-consuming nature of these methods. Grant (1950) has recently sought to overcome this difficulty by drawing or envisaging the curve from an inspection of standard and præcordial leads; his method is an approximation and no integrating apparatus is used. As the principal application of vectorcardiography is likely to be in the field of myocardial disease where conventional electrocardiography already shows a high degree of correlation with pathological findings, it would seem desirable to retain at least the degree of accuracy obtained in recording the scalar electrocardiograms.

The mathematical and physical approach that has been used by some authors to treat the fundamental problems of this subject is recognized to be of great importance; however, it may be that such esoteric analyses have also tended to slow the application of vectorcardiography to clinical medicine. It appears to us that the inherent errors in methods that on the one hand employ accurate electronic recording devices and on the other hand are concerned with such biological variables as skin resistance, tissue conductivity, chest shape, and heart position deny an elaborate mathematical analysis which, at best, gives greater definition to the subject than it actually has. Our limited experience of vectorcardiography has not enabled us to form any firm conclusions and it must be stated that our approach has been entirely empirical. 
The high cost of electronic apparatus, the difficulties of maintenance, and the time consumed in operation, all limit its use at the present time in this country. Our aim has been to develop a simple method for obtaining vectorcardiograms which might be applied to routine clinical work. In this paper we describe an apparatus by means of which the two-dimensional projections of the spatial vectorcardiogram in ea'ch plane may be drawn quickly from the appropriate leads of any reference system, and we present an analysis of the vectorcardiograms obtained by this method from 100 healthy adults.

\section{The Apparatus}

The integrating apparatus consists of a scanning table which is joined by control cables to an optical head. The two parts are mounted on the same stand. (Fig. 1).

The Scanning Table. A four-inch square of perspex sheet is mounted on a rack and pinion to form a mechanical stage, which is fixed near to the front of a wooden base so that the perspex stage may be racked in a front-to-back direction (Fig. 2). The elctrocardiogram is held to the stage by clamps.

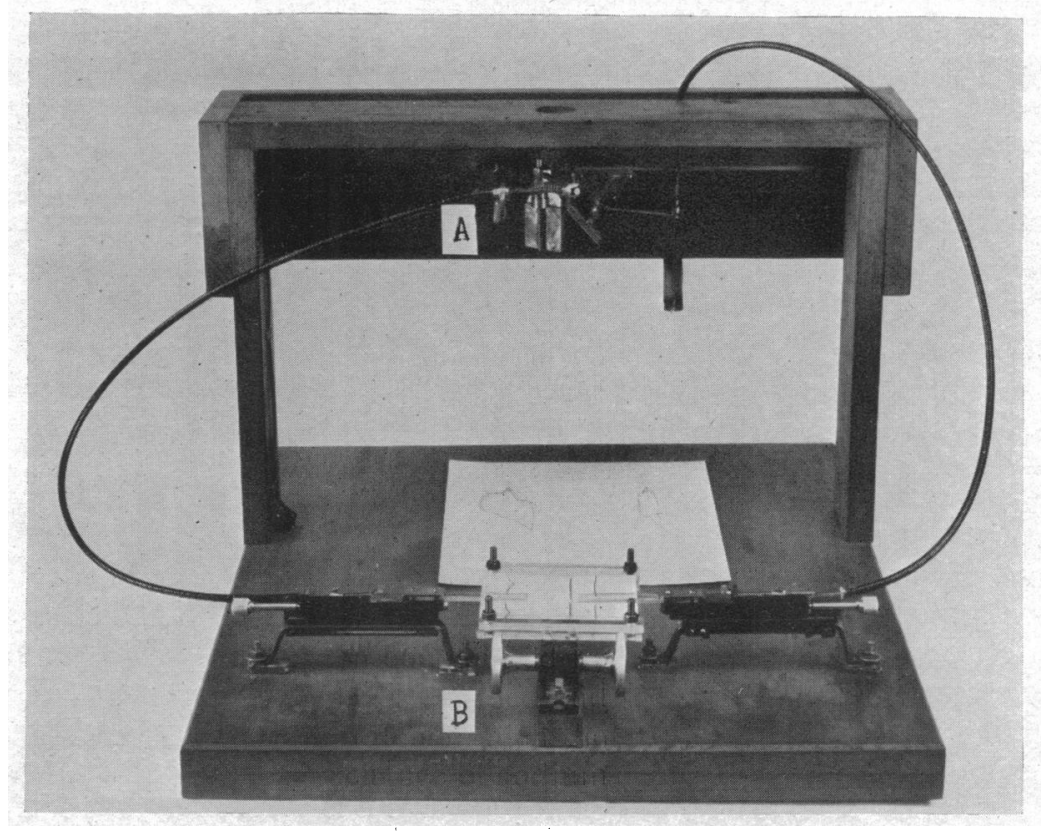

FIG. 1.-The vector drawing machine. (A) Optical head. (B) Scanning table.

Fine-adjustment mechanical stages, of the type used in the mechanical stage of a microscope are mounted on short legs secured to the base on either side of the perspex stage. Perspex fingers $(3 \times 0.5 \mathrm{in}$.) are attached to the upper movements of these stages so that they project over the scanning table and may be moved across the table from side to side by the mechanical stages. Each of the transparent fingers has a central and cross-hair line so that the voltage curve of the two underlying synchronously recorded electrocardiograms may be followed. The ends of the control cables are fixed to angle brackets which are mounted on the sides of each fine-adjustment stage. A spring-loaded screw thread with thumb screw enables a fine adjustment to be made on the cable length for the purpose of centering the light spot. 


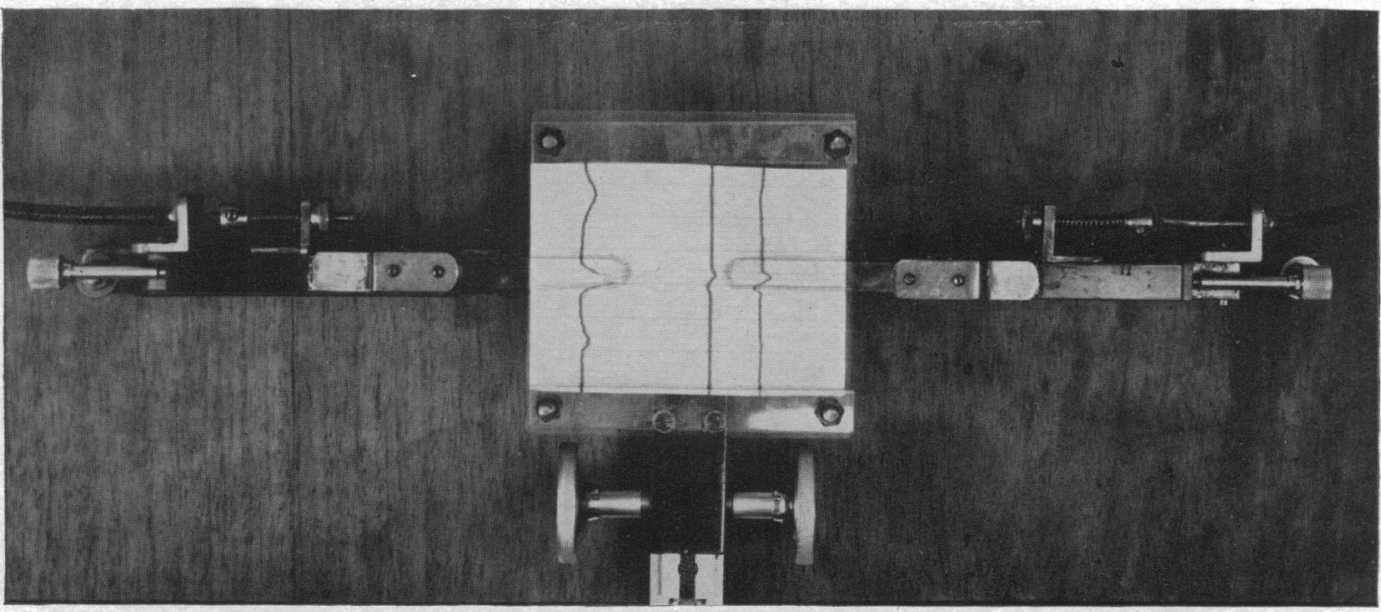

FIG. 2.-The scanning table. Perspex fingers are overlying the QRS complex of leads A and B.

The Optical Head. This is mounted on a wooden gantry twelve inches above the baseboard (Fig. 3). The light of a flashlamp bulb is reflected by means of a right-angled prism through a plano-convex lens (Fig. 4) on to the first of a pair of surface-silvered mirrors. This mirror is mounted at right angles to the gantry and may be turned by means of a $2 \cdot 5$-inch lever to which is attached the end of one of the spring-loaded control cables. Rotation of this mirror is produced by movement of the left-hand mechanical stage. The light is reflected off this first mirror on to a second mirror placed close to and at right angles to the first, and it is so arranged that the beam is reflected on to a sheet of paper placed directly below the gantry and optical head. The mounting of the second mirror is similar to the first and it is moved by the control cable from the right-hand mechanical stage. Movement of the left-hand stage will cause the light spot on the base to move in a back-to-front direction, and that of the right-hand one in a side-to-side direction.

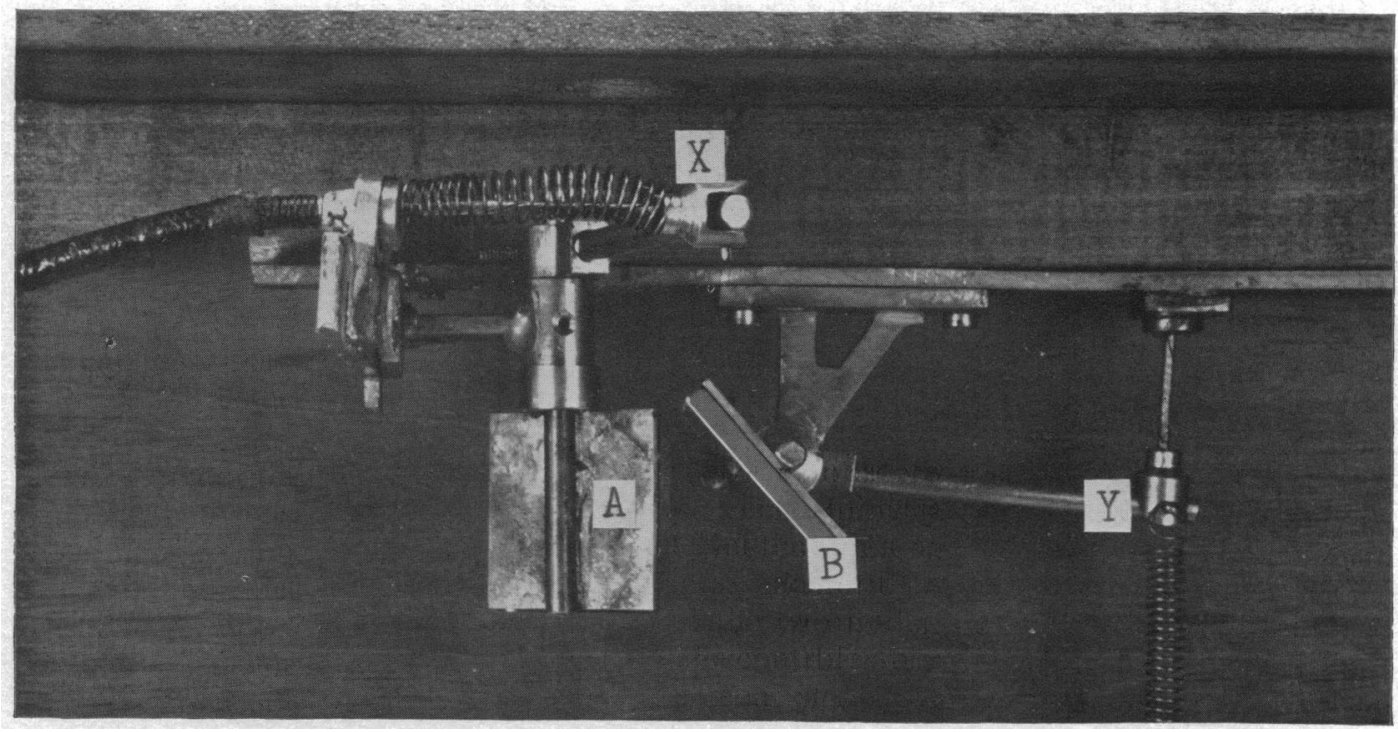

FIG. 3.-The optical head. Showing the two mirrors A and B and their respective levers $\mathrm{X}$ and $\mathrm{Y}$. 


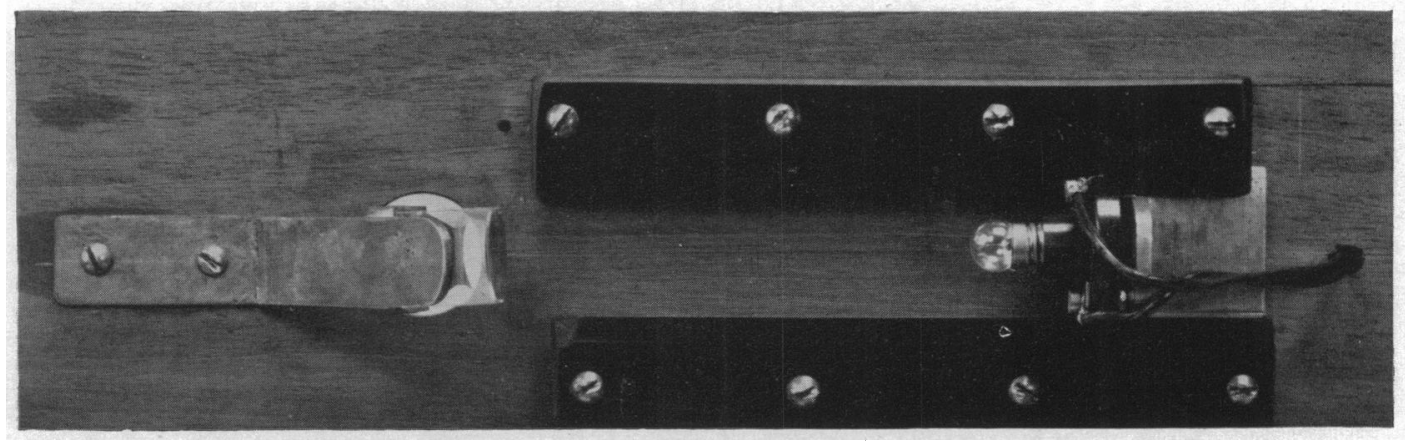

Fig. 4. - Light source and reflecting prism at the back of the optical head.

Thus it will be seen that the voltage curve of two synchronously recorded scalar electrocardiograms may be scanned by each of the perspex fingers at progressive time intervals. These fingers move the mirrors and cause a deflection of the light beam which is an integration of the two movements; this might be compared with the deflection of the electron beam by the plates of the cathoderay tube of more elaborate apparatus.

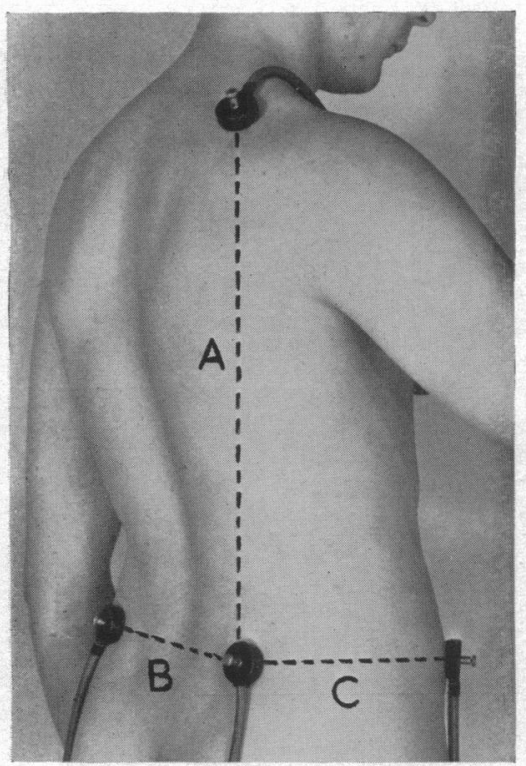

FIG. 5.-Electrode positions. A, B, and $C$ indicate the bipolar lead connections.

\section{METHOD}

(1) The Scalar Electrocardiogram. All records were taken with an Elmquist three-channel electrocardiograph which consisted of valve amplifiers with resistance capacity coupling and oil-damped mirror galvanometers, and had a frequency response almost linear to 400 cycles.

The box-shaped reference system recommended by Duchosal has been used throughout. An electrode common to the three bipolar leads to be recorded is placed over the right posterior iliac spine, and the first exploring electrode over the upper part of the right scapula; the second exploring electrode is placed over the right anterior superior iliac spine and the third over the left posterior iliac spine (Fig. 5). (These are the CR lead connections on the Elmquist machine with the right-arm connection as the common electrode.)

The simultaneous electrocardiographic tracings were made at a standardization of $1.5 \mathrm{~cm}$. to 1 millivolt, and at a paper speed of $120 \mathrm{~mm}$. a second. These factors were determined arbitrarily so that the QRS was of sufficient size to make mechanical scanning of the time intervals at 1/200th of a second simple and accurate.

We have used the apparatus described to draw the frontal, sagittal, and horizontal loops of the QRS and T complexes from the scalar electrocardiograms obtained by this method. In every case limb leads and chest leads were taken for purposes of comparison.

(2) Integration into the Vectorcardiogram. A short strip of the simultaneously recorded scalar electrocardiograms is clamped on to the scanning table so that the isoelectric lines lie in a frontto-back direction, and the tracing obtained from the vertical lead is to the left (Fig. 2). The crosswires are placed at a point on the isoelectric lines of the vertical and transverse leads (A and B). The light spot is turned on and a sheet of paper placed centrally below the optical head; on this 
sheet there are ruled vertical and horizontal lines which represent zero potential (Fig. 6). The light spot is moved by the adjusting screws on the mechanical stages until it coincides with the upper left-hand intersection of the ruled lines. The adjusting screw of the scanning table is turned so that the longitudinal hair lines on the perspex fingers coincide with the beginning of the QRS complex. The stage is advanced by an amount recorded by $1 / 200$ th of a second on the time marking of the electrocardiogram. The mechanical stages are then reset so that the cross-hair lines follow the electrocardiographic tracing to the new voltage poition. The resultant new position of the light spot which has been deviated from the isoelectric lines is recorded by a pencil mark. The whole QRS and T complex is plotted by repeating this process at time intervals of $1 / 200$ th of a second. The positions of the light spot indicated by pencil dots therefore provide time markings and a line joining them forms a loop representing the frontal projection of the spatial vectorcardiogram. Likewise, the sagittal loop is drawn by integrating the electrocardiogram from the leads $\mathrm{A}$ and $\mathrm{C}$ and the horizontal from the leads B and C. The drawing paper is arranged so that the sagittal loop is to the right of the frontal, and the horizontal below it.

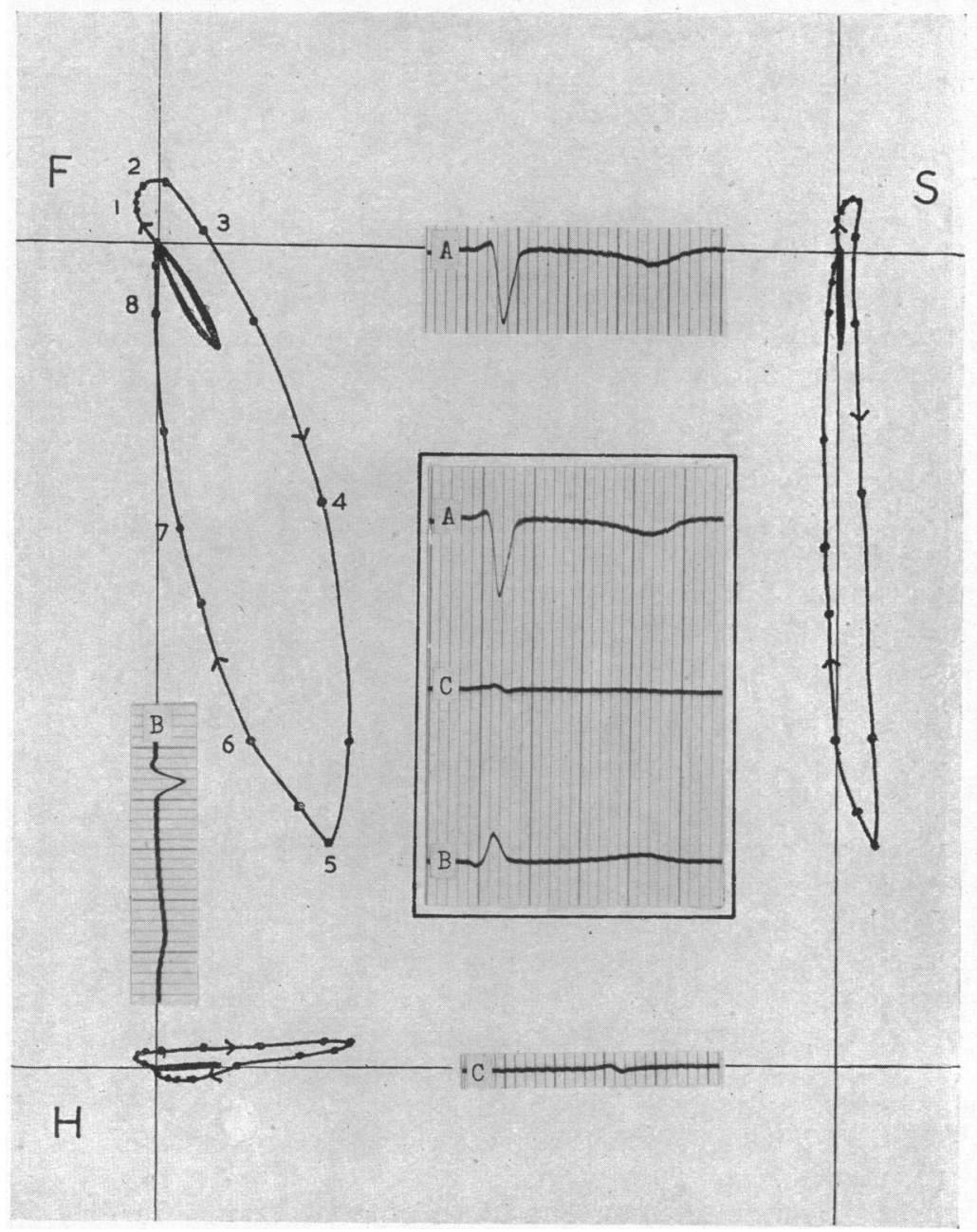

Fig. 6. -Frontal (F), sagital (S), and horizontal (H) projections of the spatial vectorcardiogram drawn from the three scalar electrocardiograms A, B, and C. The " $T$ " loop is in heavy black. The figures denote $1 / 100$ th of a second and the arrows the directions of rotation. 


\section{- Clinical. Material}

One hundred healthy adults were investigated and the majority were men; their ages were evenly distributed between 16 and 70. All were clinically examined and found to be without evidence of cardiovascular disease. Patients with a blood pressure over 160 systolic and 90 diastolic were not included. The electrocardiograms were recorded with the patient on a couch in a semirecumbent position. Most of the younger group were drawn from medical students and medical staff and the older group were selected from otherwise healthy patients who were being treated for superficial neoplastic lesions by radiotherapy.

\section{RESULTS}

The frontal, sagittal, and horizontal projections of the spatial vectorcardiogram in health were found to have characteristic features (Fig. 6 and 7). Their outline was smooth, with minor exceptions in some loops of the older age group. The rotation of the frontal loop was either in a clockwise or anti-clockwise direction. The length of the QRS loop varied from 0.06 to 0.1 sec. and the apex was reached in 0.03 to $0.05 \mathrm{sec}$. The time intervals tended to be closely spaced at the beginning and the end of the loop and spread out evenly in the middle of the tracing. In the horizontal loop several of the time interval marks were superimposed on the isoelectric line. The $T$ loops tended to rotate in the same direction and their axis lay in, or within a few degrees of the QRS loop.

The spatial vectorcardiogram was a narrow loop in the frontal view and was directed downwards and slightly to the right. The greater part was in the lower right quadrant but in some cases the return loop extended for some distance up into one of the upper quadrants. The ratio of the height of the outgoing part to the depth of the whole loop never exceeded 1:4.

In the sagittal view the tracing was also narrow and directed downwards and slightly to the right, and since the vertical component is common to the sagittal and frontal loops, extensions above and below the isoelectric were the same in both.

The horizontal spatial vectorcardiogram was narrow and small as compared with the frontal and sagittal views. Lead B is common to the frontal and horizontal loops so that the lateral extension of these loops was identical.

In the older age group, a few patients showed small kinks which were never large in comparison with the whole loop (Fig. 7C and 8B). Ten selected loops representing the limits of variation found in this series are shown in Fig. 8.

\section{COMMENTS}

We have not concerned ourselves with any of the fundamental issues of vectorcardiography or the relative merits of various spatial reference systems, but it is possible that the methods we have used might be applied to some of these problems.

The technique described has the merit of simplicity and the apparatus is inexpensive. It requires no special knowledge or experience for its operation, the three loops may be drawn quickly and the results are comparable with those obtained by other methods. The tracings made by this method are clear and considerably larger than those produced on the cathode-ray tube, and the direction of rotation is easily determined. The scalar electrocardiograms may be recorded and stored, and the loops drawn subsequently; the individual QRS and T complexes may be drawn separately and remain quite distinct. If desired, the scalar electrocardiogram may be photographed at faster speeds and at greater amplification.

The error introduced by the distance between the mirrors and consequent difference in magnification of two scalar components is corrected by one of the mirror levers being slightly longer than the other. Any distortion produced at the edges of the field is small because the distance from the base to the optical head is large in relation to the size of the vectorcardiogram. We have found that 

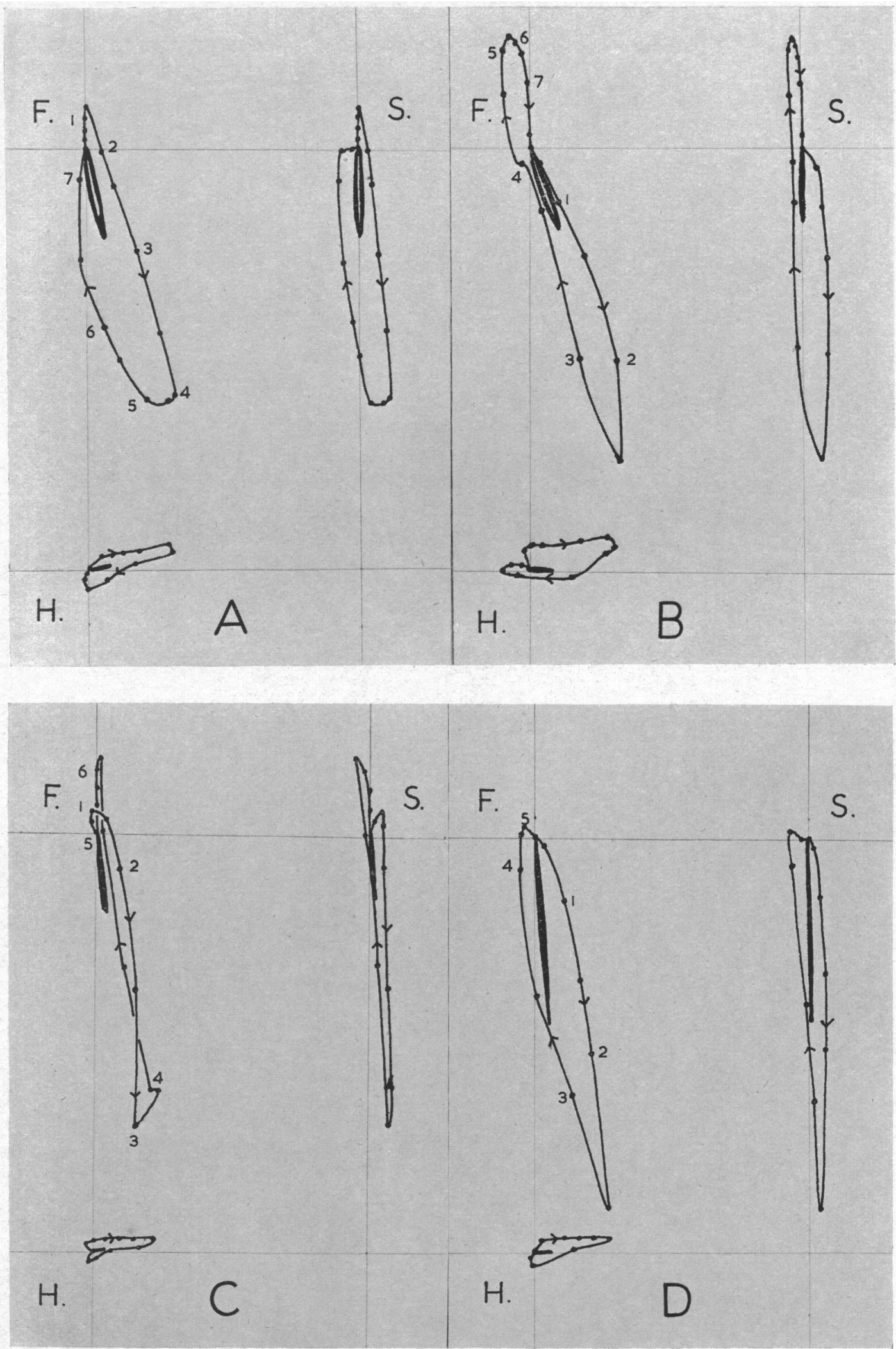

Fig. 7.-Examples of the vectorcardiogram in health: (A) shows a small upward projection on the outgoing part of the loop; (B) has the upward component on the returning limb; (C) shows a kink at 4/100ths of a second; and (D) a smooth loop with no irregularities. 


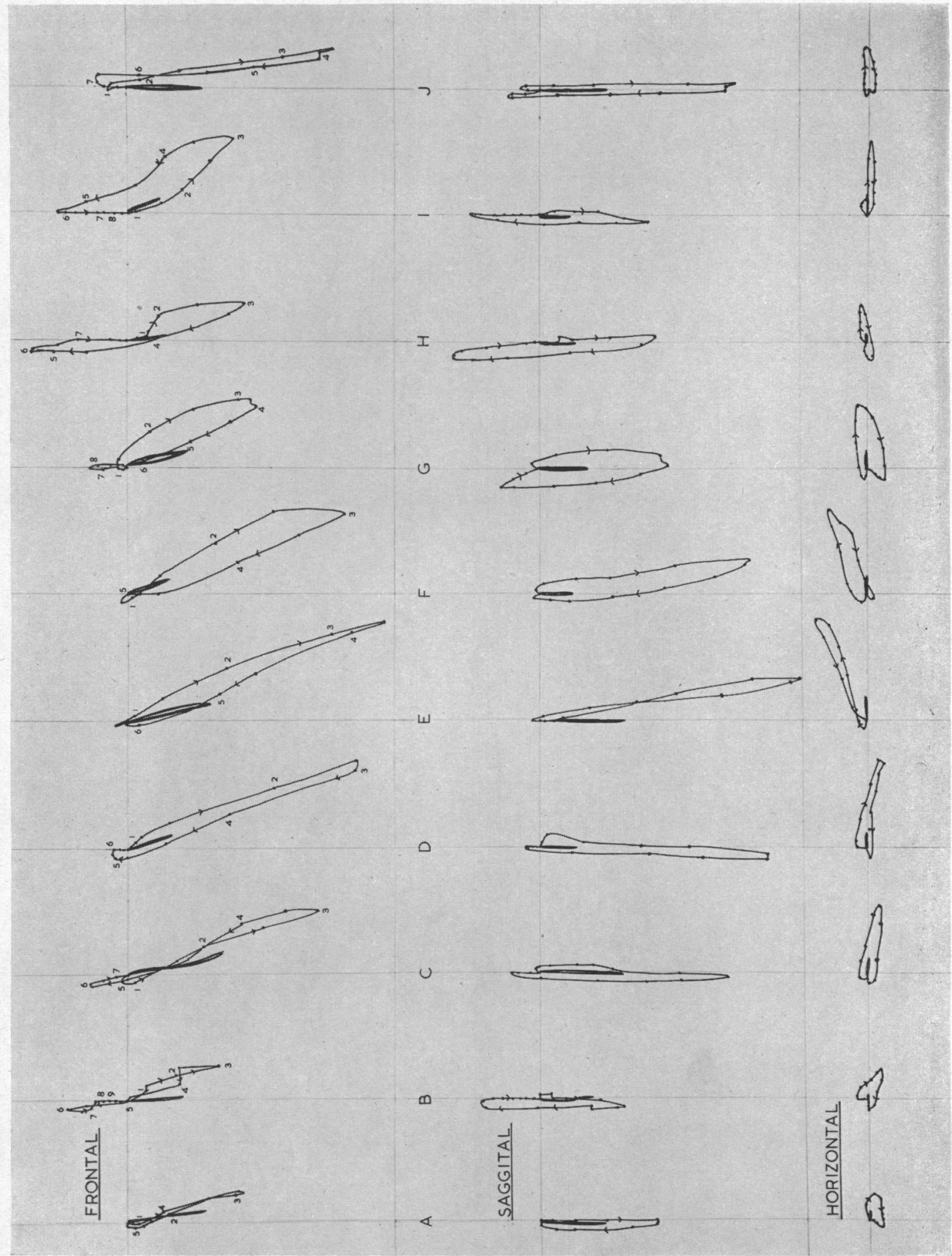


the spring loading of the control cables ensures that there is no backlash between the scanning fingers and the light spot.

The 100 healthy adults examined showed a variety of patterns, but these fell within limits that were not difficult to define. The patterns were similar to those obtained by Duchosal and Sulzer (1949). when allowance was made for different polarity and direction of viewing. The normal patterns obtained from the tetrahedral reference system and illustrated by Conway et al. (1949) were also similar to those seen in our series. We have not constructed three-dimensional models from the plane drawings of the vectorcardiogram; apart from their value for demonstration purposes, such models have not appeared to us to give information that could not readily be obtained from the drawings.

The loops were characterized by a smooth contour but a few from the older age groups showed slight irregularities or kinks although these patients were normal clinically. It is, perhaps, not irrelevant speculation to relate this loss of smoothness to degenerative changes in the myocardium.

\section{SUMMARY}

A new method of integrating scalar electrocardiograms in two planes is described. The apparatus relies on mechanical scanning of the electrocardiograms and their optical integration into a vectorcardiogram.

One hundred healthy subjects have been examined, using a parallelepiped reference system, and the scalar electrocardiograms thereby obtained have been integrated into frontal, sagittal and horizontal loops.

The vectorcardiograms drawn by this method resemble those produced by elaborate electronic methods. The loops, which fall into a well-defined range in position, shape and time relations, have been described.

We are indebted to Dr. J. P. D. Mounsey for help and criticism.

\section{REFERENCES}

Conway, J. P., Cronvitch, J. A., and Burch, G. E. (1949). Amer. Heart J., 38, 537.

Duchosal, P. W., and Sulzer, R. (1949). La Vectorcardiographie, S. Karger.

Grant, R. P. (1950). Circulation, 2, 676.

Mann, H. (1920). Arch. intern. Med., 25, 283.

- (1938). Amer. Heart J., 15, 681.

Schellong, F. (1936). Verhandl. deutsch. Gesellsch. inn. Med., 48, 288.

Wilson, F. N., and Johnston, F. D. (1938). Amer. Heart J., 16, 14. 